\title{
RFID READER TESTING KIT FOR AUTONOMOUS AIRSHIP
}

\author{
POSPISILIK, M[artin] \& ADAMEK, M[ilan]
}

\begin{abstract}
Within the framework of the Autonomous airship project that is being developed at Tomas Bata University in Zlin, an RFID testing kit has been created in order to test the possibility of applying the RFID technology at airship locating inside an enclosed hall. In this paper the hardware design of such testing kit is described. The kit should serve for processing various tests of RFID antennas and controlling algorithms

Keywords:RFID, autonomous monitoring system, reader, hardware design
\end{abstract}

\section{INTRODUCTION}

This unit has been developed as a part of an Autonomous Monitoring System which is a project that is being solved at Tomas Bata University dealing with a problem of attaching a small airship with some monitoring equipment. This airship is then capable of an independent operation inside an enclosed hall, monitoring its neighbourhood. The RFID tags displaced around the hall surface are intended to determine the airship position as it is not convenient to use GPS technology, because the operating area can be too small compared to the basic accuracy of the GPS technology.

The basic orientation of the airship is ensured by employing the inertial positioning system. When the point from which the airship starts is known, by measuring of time, speed and direction its trajectory can be monitored and foretold. The main advantage of this positioning system consists in the fact that it shows considerable cumulative error that increases in time. In order this error was suppressed, the RFID tags are displaced on the airship's trajectory, giving the information on whether at the current position the prescribed trajectory is followed or not. Provided more RFID tags are displaced around one checkpoint, one of them gives the feedback on the proper trajectory while the others give feedback on the necessary corrections of the trajectory.

In order the RFID technology worked properly, the tags must be detected at the minimum distance of $1 \mathrm{~m}$ from the antenna. This is the reason why AS3910 RFID reader was employed. Because of its $1 \mathrm{~W}$ output power and operating frequency of $13.56 \mathrm{MHz}$ the minimum distance should be obtained. The reader was implemented on one printed circuit board together with a Freescale MC9S08SE4 microcontroller that is intended for being used as a CPU of the whole autonomous monitoring system. The antenna and the voltage regulator can be connected as separated modules in order several types of antennas could be tested as well as different types of voltage regulators, especially the ones working in switched mode, producing a considerable amount of electromagnetic interferences.

\section{PHILOSOPHY}

The block diagram of the hardware kit is depicted in the Fig. 1. It can be seen that it consists of three PCBs - a motherboard that includes RFID reader and the microcontroller, a voltage regulator and an antenna.Moreover, the kit is provided with a BDM interface that enables the user to load his own software into the microcontroller and its debugging as well. Different voltage regulators can be applied in order it was proven how sensitive the circuit is to their interference.

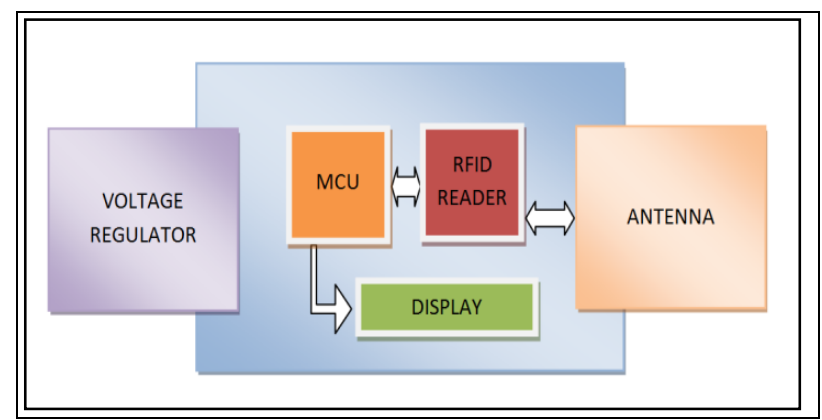

Fig. 1. Hardware kit block diagram

\section{CONSTRUCTION}

In this chapter there is a description of the circuitry. All the modules depicted in Fig. 1. are described separately.

\subsection{Linear voltage regulator}

The linear voltage regulator is based on a monolithic stabilizer LM317T. The input supply voltage is supposed to lie between 5 and $12 \mathrm{~V}$. For the maximum current load, that is expected to be around $0.4 \mathrm{~A}$, there is a need of a small heatsink that dissipates at least $3.5 \mathrm{~W}$. The feedback is designed according to (1) in order the voltage of $3.5 \mathrm{~V}$ was reached at the output of the regulator, considering the additive current of $100 \mu \mathrm{A}$ that is being emmited from the reference input of the stabilizer.

$$
U_{\text {OUT }}=1.25 \cdot\left(1+\frac{R 102}{R 101}\right)+I_{A D J} \cdot R 102[V]
$$

The meanings of the parameters are as follows:

- $\mathrm{U}_{\text {OUT }}$ - output voltage [V],

- R101, R102 - resistance of resistors used in the feedback [ $\Omega]$, 
- $\mathrm{I}_{\mathrm{ADJ}}$ - additive current emmited by the ADJ pin of the stabilizer.

The schematic of the linear regulator is depicted in Fig. 2. The linear voltage regulator is connected to the main board via the SV101 connector.

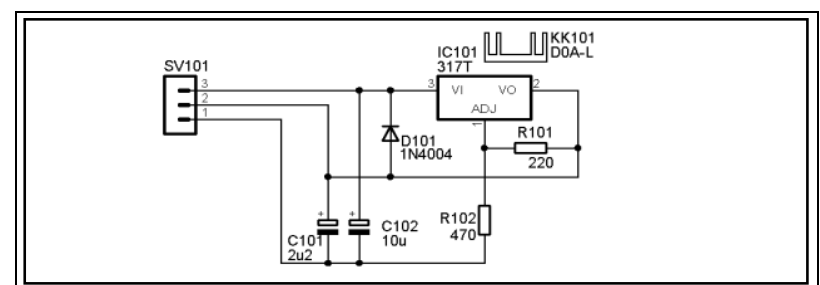

Fig. 2.Linear voltage regulator

\subsection{Switching mode voltage regulator}

The switching-mode voltage regulator is based on MC34063 chip and has been constructed in order to prove how the reader is capable of operation near the electromagnetic interference source. Circuit topology and device values were derived according to the MC34063 manufacturer's hints. The schematic of the circuit can bee seen in Fig. 3.

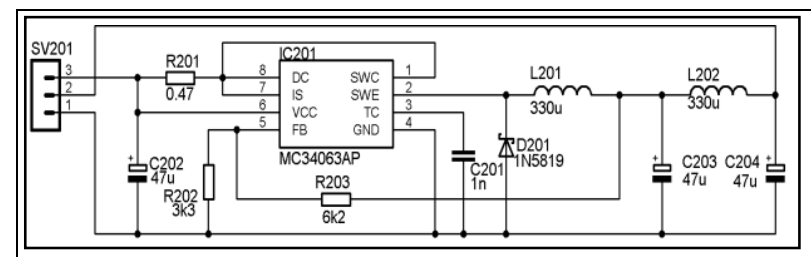

Fig. 3. Switching-mode voltage regulator

The boundary parameters were considered as follows:

- input voltage from 5 to $15 \mathrm{~V}$,

- maximum output current up to $400 \mathrm{~mA}$,

- output voltage $3.3 \mathrm{~V}$,

- minimum operating frequency $30 \mathrm{kHz}$.

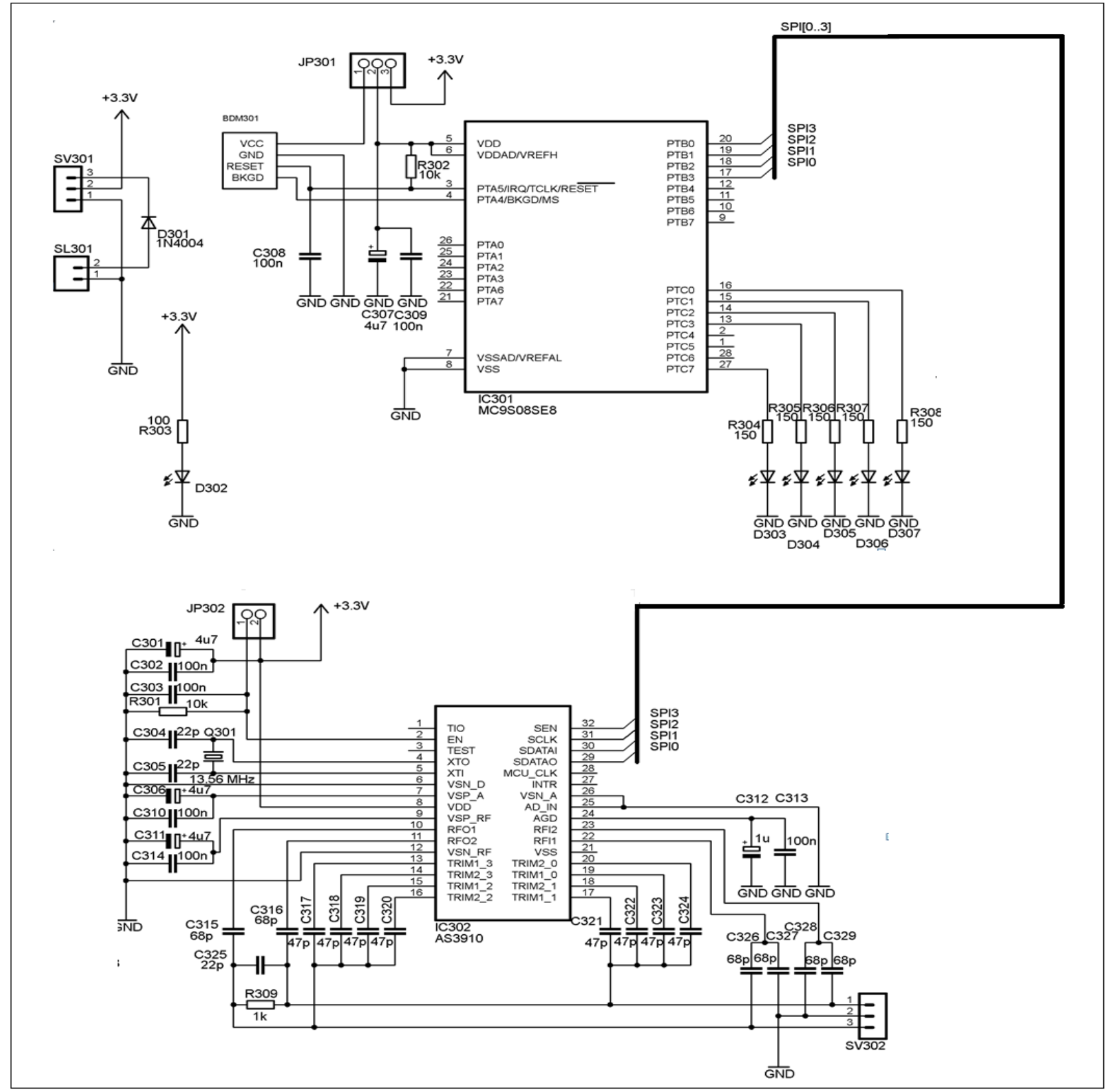

Fig. 4 Main board schematics 
Both inductors, L201 and L202 are of toroid type. The D201 diode must be a fast Schottky type. All other devices are the standart ones. The regulator is connected to the main board via the SV101 connector.

The switching-mode voltage regulator based on the MC34063 chip operates asynchronously. Its regulation operates on the basis of omitting of periods if the full output power is not required. Therefore it can be considered as an ideal source of interferences.

\subsection{Main board}

Two main devices of the RFID reader are implemented on the main board. The RFID reader chip AS3910 and the MCU Freescale MC9S08SE8. The RFID reader chip was chosen for its capability of reaching up to $1 \mathrm{~W}$ transmitting power that enables the reader to achieve more than $1 \mathrm{~m}$ reading distance. Unfortunatelly, the chip is only available in QFN32 package which makes it very difficult to solder manually without special equipment. The MCU is of the same type as the one supposed to be employed as a central MCU of the whole airship which is convenient for creating the appropriate software.

Apart from the two main devices, there are also 6 LED indicators, connectors for connecting power supply, voltage regulator, antenna and external BDM programmer as well as other devices necessary for the proper function of the whole circuit. The schematic of the main board can be seen in Fig. 4 .

The communication between the microcontroller and the RFID reader is ensured via the SPI bus. The microcontroller sends commands to the reader and waits for its response. The kit supports up to 4 tags the addresses of which are stored in the memory of the MCU. If the microcontroller asks the reader to send the address of a close tag and the appropriate tag is detected, it can be indicated by one of the LEDs D304, D305, D306 or D307. The D303 LED is used for indication of error states, for example if reader is not capable of calibrating the antenna. The D302 LED indicates that the power is on.

The connector SL301 serves for connecting the power supply. The appropriate voltage regulator must be connected to the SV301 slot. The D301 diode protects the circuitry from connecting the power supply source with bad polarity. The programming device can be connected to BDM301 connector. Conventional Freescale BDM interface is used. By the JP301 jumper the user can select whether the MCU is supplied with the programming device or with the main board supply net. This enables programming of the MCU without connecting the main board to the power supply source. Moreover, for the purposes of SW testing, the operation of the RFID reader can be disabled by disconnecting the jumper JP302.

For the proper function of the RFID reader accurate clock frequency source is needed. This is ensured by employing the Q301 crystal.

The antenna is connected to SV302 slot. It is driven via the capacitors $\mathrm{C} 315$ and $\mathrm{C} 316$. The set of capacitors from $\mathrm{C} 317$ to $\mathrm{C} 324$ serves for automatic antenna calibration. The calibration can be run by the appropriate command sent by the MCU and its result is then reported back to the MCU.

\subsection{Antenna}

The antenna is made in a form of a double-sided spiral coil on a printed circuit board. As an auxiliary PCB it can be connected to the appropriate slot on the main board. Its shape was derived from other antennas used in RFID systems. This is why it is supposed to be of the same parameters.

\section{SOFTWARE}

The flowchart of the software implemented in the microcontroller is depicted below.

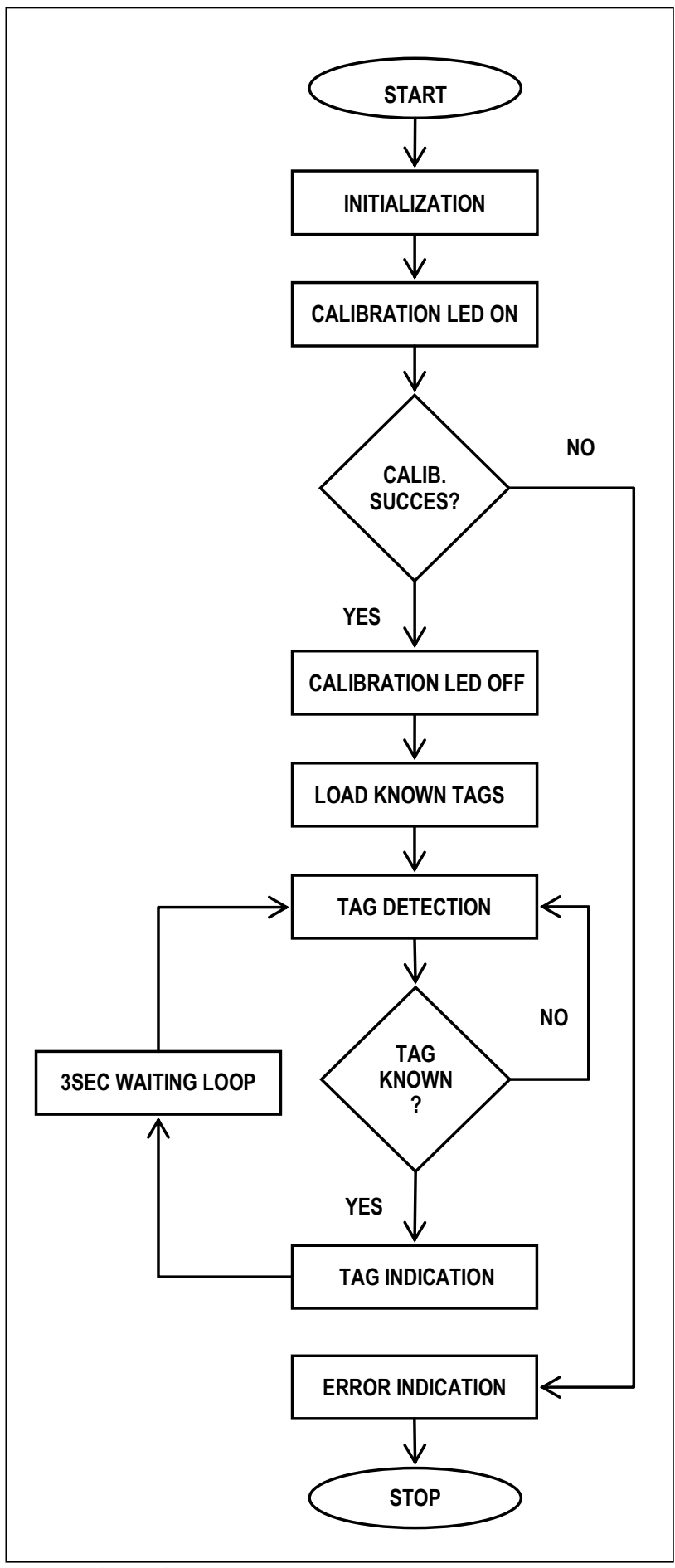

Fig. 5. Software flowchart 
The communication between the microcontroller IC301 and the RFID reader IC302 is ensured by the SPI bus. According to its datasheet, the RFID reader expects commands in the form of bit sequences and consequently it sends back the requested data. Moreover, its modes of operacion can also be switched by means of the commands sent by the microcontroller. The example of the communication protocol of the microcontroller and the RFID reader is depicted in Fig. 5. The communication is always controlled by the microcontroller by setting the SEN lead of the bus to the $\mathrm{H}$ level. It also produces clock signal on the SCLK bus.The data transfer operated on the leads SDATAO and SDATAI.

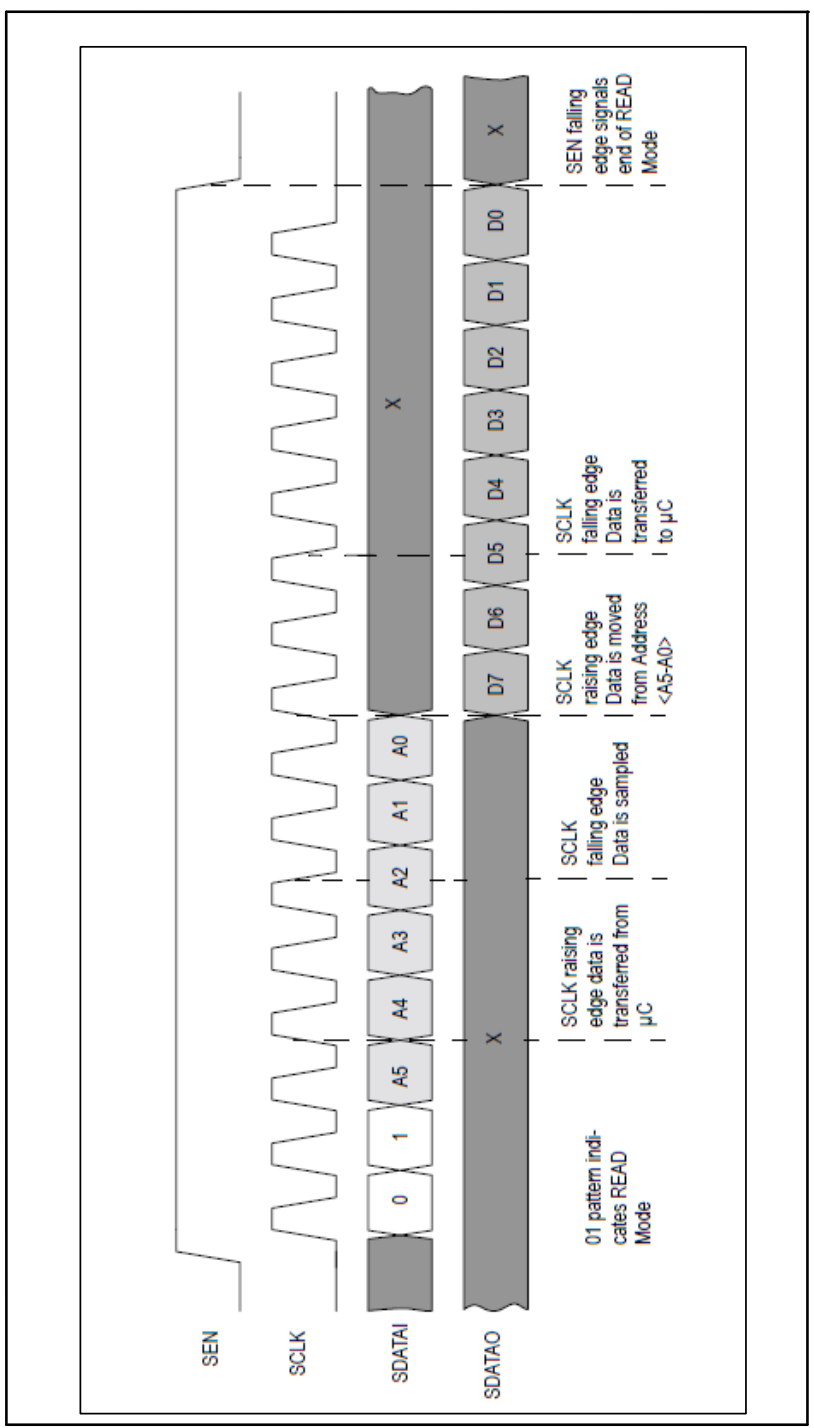

Fig. 6. Example of communication between the microcontroller and the RFID readed

The sequence of the commands used for initialization of the reader is enlisted in Tab. 1.

Programming of the microcontroller has been processed according to the information in its datasheet [5] by means of the $C$ language, including pieces of code written in assembler. All the information of the possibilities of the RFID reader and the commands it recognizes can be found in the proper documentation [6]. Code Warior Development Studio software by Freescale was utilized to create the code.

\begin{tabular}{|c|c|l|}
\hline Instruction & Instruction name & \multicolumn{1}{c|}{ Comment } \\
\hline 000001 & SET DEFAULT & $\begin{array}{l}\text { Initialization to the } \\
\text { default state }\end{array}$ \\
\hline 000010 & CLEAR & $\begin{array}{l}\text { Interrupts the whole } \\
\text { operation and clears the } \\
\text { output buffer }\end{array}$ \\
\hline 010110 & $\begin{array}{c}\text { ADJUST } \\
\text { REGULATORS }\end{array}$ & $\begin{array}{l}\text { Internal voltage sources } \\
\text { automated calibration }\end{array}$ \\
\hline 011000 & $\begin{array}{c}\text { CALIBRATE } \\
\text { ANTENNA }\end{array}$ & $\begin{array}{l}\text { Antenna calibration by } \\
\text { means of the TRIM } \\
\text { capacitors }\end{array}$ \\
\hline 011001 & $\begin{array}{c}\text { CHECK } \\
\text { ANTENNA } \\
\text { RESONANCE }\end{array}$ & $\begin{array}{l}\text { The state of antenna } \\
\text { calibration is loaded to } \\
\text { the 0E register }\end{array}$ \\
\hline
\end{tabular}

Tab. 1 RFID reader initialization sequence

The RFID reader supports the following modes of operation:

- WRITE,

- READ,

- FIFO Load,

- FIFO Read,

- COMMAND.

The mode selection is determined by the first bits set by the microcontroller after setting the SEN lead to the $\mathrm{H}$ level. The mode selection is needed, because the RFID reader contains its internal registers and by setting their values, its operation can be controlled.

\section{CONCLUSION}

A hardware design of an RFID reader testing kit is described in this paper. This kit has been made and currently it is a subject to undergo operational tests and software debugging. It is supposed to be integrated as a part of the Autonomous monitoring system.

The purpose of the reader is to utilize the RFID tags in suppressing of the cumulative error that occurs in case the inertial positioning system is employed.

\section{ACKNOWLEDGEMENT}

Thispaperissupported by theInternal Grant Agencyat TBU in Zlin, project No. IGA/45/FAI/10/D and by theEuropeanRegionalDevelopmentFundundertheproject CEBIA-Tech No. CZ.1.05/2.1.00/03.0089.

\section{REFERENCES}

[1] Sommerova, M. (2008). Introduction to RFID technology [Zaklady RFID technologii][online]. Ostrava: VSB. 32 p. Available from: http://rfid.vsb.cz/miranda2/export/sites-

root/rfid/cd/okruhy/informace/RFID_pro_Logistickou_akademii.pd $\mathrm{f}$

[2] Brown, D. E. (2007). RFID implementation. New York: McGrawHill, 466 p., ISBN-0-07-226324-5

[3] Pospisilik, M.; Adamek, M. (2010). Autonomous Airship, 9th International Conference PROCESS CONTROL 2010, pp. C075a1 - C075a-6, Kouty nad Desnou: 2010

[4] http://www.freescale.com(2010) - Freescale microcontrollers, Accessed on 10-08-2012

[5] http://www.austriamicrosystems.com(2011) - RFID reader AS3910 datasheet, application information, Accessed on: 10-082012 